\title{
Mejoras en el diseño y uso de LED para medir la constante $h$ de Planck
}

\author{
Luis Vargas ${ }^{1}$ Y Maximino Suazo ${ }^{2}$ \\ ${ }^{1}$ Escuela de Física - UNAH , mail: levargashn@gmail.com \\ ${ }^{2}$ Escuela de Física - UNAH , mail: suazomaximino@yahoo.com
}

Recibido: 28 de febrero del 2017 / Aceptado: 30 de abril del 2017

\begin{abstract}
Resumen
Calculating the PLANCK constant has been a discussed experiment by many researchers within the academic and experimental field, where the focus has been finding an optimal outcome. In this paper, we present different approaches and methodologies, analyzing diverse points of view for each experiment, considering the use of LED and $R C$ circuits. These are discussed with the purpose of finding a design and/or methodologies that allow more precise and viable calculus. Our design showed a value for the PLANCK constant of $(6.7 \pm 0.8) \times 10^{-34} \mathrm{~J} \cdot \mathrm{s}$, which is both accurate value and a good approximation to the theorical value.
\end{abstract}

Keywords: Planck, Design, LED, RC

El cálculo de la constante de PLANCK ha sido un experimento discutido por muchos catedráticos en el ámbito académico y experimental en donde la búsqueda de un resultado fiable ha sido el enfoque primordial. En este artículo se presentan diferentes enfoques y metodologías analizadas desde diversos puntos de vista para cada experimento, considerando el uso de LED y circuitos RC. Estos se discuten para encontrar un diseño y/o metodologías que permitan cálculos más precisos y confiables. El diseño elaborado presentó un resultado para el valor de la constante de PLANCK de $(6.7 \pm 0.8) \times 10^{-34} \mathrm{~J} \cdot \mathrm{s}$, el cual es un valor exacto y con una buena aproximación al valor teórico.

Palabras clave: Planck, Diseño, LED, RC

\section{INTRODUCCIÓN}

$\mathrm{E}$ N 1889, Max Planck introdujo el concepto de "cuanto de acción" (i.e. Constante de PlanCK) en un intento de explicar la distribución espectral de la radiación del cuerpo negro, siendo este un fundamento para la teoría cuántica [7]. La constante fue primeramente descrita como un valor de proporción que relaciona la energía de un fotón y la frecuencia asociada a las ondas electromagnéticas. El comportamiento de las ondas electromagnéticas asociadas a este cuanto de acción se estudia bajo diversos conceptos que exploran sus relaciones y propiedades físicas. Una forma simple de expresar esta relación entre la energía y la frecuencia es llamada la relación de Planck o la ecuación de Planck-Einstein (1).

$$
E=h f
$$

La constante de Planck, ha sido calculada haciendo uso de diversos métodos y procedimientos experimentales, los cuales se han vuelto moderadamente más complejos a través del tiempo debido a aportaciones de estudiantes y catedráticos [9] [10, 2] [5] [12] quienes han presentado resultados favorables para la estimación del valor de la constante con bajas incertidumbres. La mejora en los resultados se logró realizando variaciones en el diseño experimental e introduciendo nuevas variables en los cálculos. Las variaciones en el diseño experimental son el producto de cambios en instrumentos y metodologías de medición, que han proporcionado resultados más exactos y bajas incertidumbres (i.e. $<5 \%$ ).

En algunos experimentos encaminados a medir la constante de Planck, utilizando materiales de bajo costo, se toman valores de ciertas variables de la información proporcionada por el fabricante de los instrumentos de medición, mismos que no son corroborados por los experimentadores. En consecuencia, se corre el riesgo de que los resultados experimentales no correspondan con los valores esperados, ni tengan la precisión y exactitud deseadas. Esos experimentos, proporcionan resultados con incertidumbres porcentuales relativamente altas (e.g. > $10 \%)$. Por lo tanto, se ha considerado mejorar el diseño experimental implementando nuevos cambios que aseguren un resultado con incertidumbres más bajas $(<10 \%)$.

En este artículo se presenta una nueva alternativa basada en estudios previos; se propone la medición de la longitud de onda para cada LED de dos maneras, utilizando y sin utilizar los valores predeterminados. Una 
de las finalidades es identificar puntos de compatibilidad entre las metodologías previas definiendo así procesos y condiciones que determinan la fiabilidad del diseño y ejecución del experimento.

\section{OBSERVACIONES DEL DISEÑO EXPERIMENTAL}

En el diseño experimental que se realiza para calcular la constante $h$ de PLANCK, el uso de LED es fundamental ya que facilita la diversidad de emisores de luz a un bajo costo [1]. Se escoge el LED ya que es un semiconductor que emite luz debido a su capacidad de electroluminiscencia, la cual emite luz debido al aumento de energía en la banda del semiconductor.

En estudios anteriores [9] 10] [2] 5] 8] [12] el proceso de medición de la constante de PLANCK se ha basado en el uso de un circuito RC cuyo diseño busca la determinación del mínimo voltaje que se necesita para lograr la emisión de luz por parte del LED. Este tipo de diseño considera al LED como una resistencia que al ser parte de un circuito que carece de una fuente de poder disminuirá el voltaje almacenado en el capacitor. El enfoque de este modelo es llevar al capacitor hasta donde no posea suficiente voltaje para alimentar el LED, llegando al momento en donde este deje de emitir luz. En ese momento la descarga del capacitor se detendrá manteniendo entre sus terminales un voltaje de valor fijo, mismo que equivale al voltaje mínimo necesario para excitar el semiconductor, el cual se encuentra en la frontera del nivel de la banda, antes que este la logre superar y haga que el LED emita luz [12. Estos estudios [9] 10] [2] [5] [12], al concentrarse en el cálculo del valor del voltaje mínimo como un punto clave del experimento han realizado diversas combinaciones de montajes, utilizando distintos componentes. De igual forma han asumido valores pre-establecidos o conocidos basados en el tipo LED y el color observable que este emite. Estas asunciones han permitido utilizar los valores de longitudes de ondas proporcionados por los fabricantes de los LED, los cuales se encuentran publicados en tablas, generalmente sin estar acompañados por la incertidumbre absoluta respectiva. Cabe mencionar que no todos los fabricantes divulgan la información de esa forma, algunos proporcionan la incertidumbre absoluta o porcentual.

Asumir los valores de longitud de onda para cada LED conlleva a obviar los errores relacionados a la calidad del material. Este tipo de asunciones puede ser válida para experimentos que utilizan materiales diseñados específicamente para el uso experimental y no comercial, en donde la fabricación ha seguido una inspección más rigurosa.

En experimentos de bajo costo, los cuales se desarrollan en instituciones educativas como parte de metodologías de enseñanza o como demostraciones académicas, se hacen ciertas asunciones necesarias para realizar la demostración. No obstante, se debe tener en cuenta que los instrumentos utilizados generalmente son producidos en masa debido a su bajo costo por lo que pueden poseer errores de fabricación que crean discrepancias entre los valores especificados en las tablas proporcionadas por el fabricante y los valores reales de cada LED.

Con el propósito de tener un valor más confiable de la longitud de onda, y no simplemente asumir el valor de tablas proporcionadas por el fabricante, se ha diseñado un montaje para medir la longitud de onda de cada LED modificando experimentos previos [9] [10] [2][5] [8][12] al incluir ideas que posiblemente mejoren el diseño [11].

\section{Metodología}

\section{A. Medición de la Longitud de Onda}

El montaje del experimento para determinar la longitud de onda depende de dos elementos clave, un LED que debe emitir un haz de luz en dirección a una rejilla de difracción que deberá tener un alto número de líneas o separaciones por centímetro. La combinación de ambos elementos es necesaria para dar lugar al fenómeno conocido como difracción e interferencia de la luz [11] [3]. Este fenómeno divide el haz de luz en varios nodos con separaciones equidistantes a ambos extremos de la dirección original [3] (Fig. 1). La simetría que se genera sigue la dirección del haz de luz, el cual puede ser observado al proyectarse sobre una pantalla de observación.

El uso de este fenómeno hace posible determinar la longitud de onda del haz de luz emitido por el LED basando los cálculos en las relaciones geométricas del montaje y del fenómeno en cuestión. Este mismo es utilizado para realizar la medición de la longitud de onda de cada LED que emite luz dentro del espectro visible. Este procedimiento se utiliza como una alternativa al uso de las tablas proporcionadas por los fabricantes de LED. Lo cual puede mejorar los resultados del cálculo de la constante $h$ de PLANCK, y a la vez generar incertidumbres más confiables que las obtenidas de los montajes realizados con materiales de bajo costo, en donde se asumen los valores de las longitudes de onda.

El análisis del fenómeno de difracción producido, se caracteriza en términos de las propiedades físicas de la onda y de la rejilla. El espaciamiento entre las líneas de la rejilla es un parámentro importante de medición, ya que cuando el haz de luz pasa por las rendijas, ésta se divide en varias ondas que se superponen en puntos específicos del espacio. Al momento de encontrarse estas ondas unas con otras, éstas entran en interferencia. La interferencia producida entre las ondas se logra observar al ser proyectada sobre una superficie robusta en donde la interferencia constructiva será la que produzca nodos visibles. La expresión mostrada en la ecuación (2) define una relación líneal para este fenómeno en donde se toman 
en cuenta la cantidad de nodos formados por interferencia constructiva. A la variable que define los nodos de interferencia constructiva se le conoce como el orden de interferencia . Asimismo, la expresión considera otros factores mencionados previamente como ser la longitud de onda $\lambda$ y el espaciamiento d de la rejilla.

$$
d \sin (\theta)=m \lambda
$$

El análisis del fenómeno difracción considera la geometría del mismo estableciendo no sólo un orden de interferencia, sino también para cada nodo de interferencia constructiva que se proyecta desde la rejilla se determina un ángulo de deflexión $\theta$, el cual se mide como el ángulo de separación entre el orden cero $(m=0)$ y el orden que se esté observando (Fig. 1).

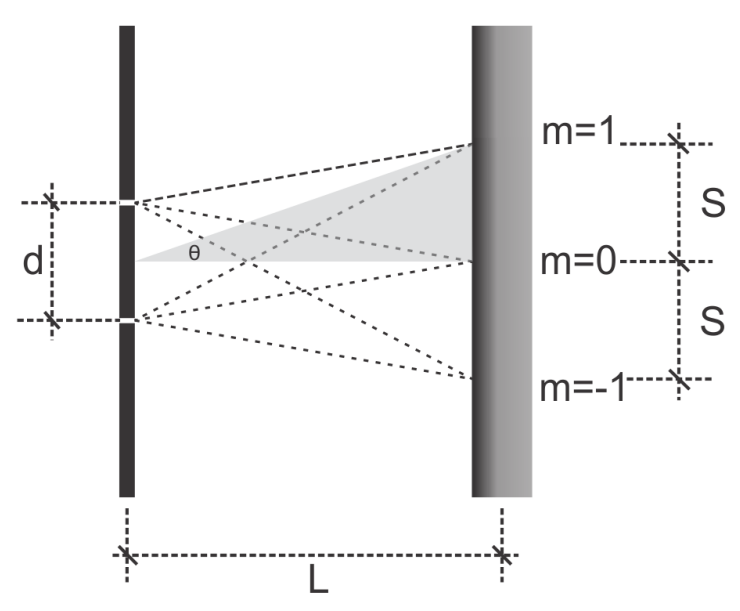

Figura 1: Ilustración de la geometría involucrada en el fenómeno de difracción con sus respectivas variables instrumentales y medibles.

Debido a que el ángulo de deflexión es un parámetro que resulta difícil determinar mediante mediciones directas, se hace uso de relaciones geométricas en donde la separación $\mathrm{S}$ entre los puntos de interferencia constructiva se define para mejorar la incertidumbre de los cálculos y facilitar las mediciones, tal y como se ha hecho en estudios anteriores (Fig. 1). Las relaciones geométricas involucradas en la proyección de los nodos implican mayor interés en el diseño de las dimensiones del montaje, en donde la distancia entre la rejilla y la pantalla (o superficie) L, donde se proyectan los nodos, es un valor clave.

$\mathrm{Al}$ establecer todas las relaciones geométricas del montaje utilizado se logra una expresión para la longitud de onda (3) que se basa sólo en los medibles del experimento. Esto satisface el enfoque de un experimento de bajo costo en donde las variables sean medibles y manejables.

$$
\lambda=\frac{d \sin (-1) \tan \left(\frac{S}{L}\right)}{m}
$$

\section{B. Medición del Voltaje de emisión}

El circuito RC analizado y trabajado en esta investigación es similar a estudios previos [9] 10] [2] [5] [8], haciendo uso de esquemas en donde se determina el voltaje mínimo necesario para que el LED emita luz. El capacitor llegará a un voltaje mínimo cuando se descargue a tal punto que el LED deja de conducir la corriente y el circuito queda abierto. Este caso se analiza como un decaimiento (4) el cual depende de las componentes del montaje (i.e. el capacitor $C$ y el LED como resistencia $R$ ). A diferencia de otros autores 9 que utilizan componentes extras para realizar un análisis de distribución de la corriente en el circuito, este estudio se enfocó en los voltajes involucrados en el circuito (i.e. el voltaje inicial que posee el capacitor $V_{0}$ y el voltaje terminal o remanente $V_{T}$ que tendrá cuando el LED deje de emitir luz). Con esto se logra un análisis más simple que depende de un solo instrumento que posee gran precisión (Fig. 2).

$$
V_{c}(t)=V_{o} \exp \left[-\frac{t}{R C}\right]+V_{T}
$$

Al tomar en cuenta la teoría que fundamenta la ecuación de PLANCK (1), que muestra la relación entre la energía y la frecuencia de una onda, se analiza la energía necesaria para que un electrón deje su órbita y se irradie un fotón o luz, lo cual es el principio del funcionamiento de los LED. Por lo tanto la energía necesaria para que un LED irradie luz se infiere como el trabajo mínimo que se debe realizar para mover un electrón de su órbita, en donde el trabajo necesario se puede expresar como la diferencia de voltaje necesaria para que un electrón se desplace (5).

$$
W=q \Delta V \rightarrow e V_{T}=E
$$

Al tomar la ecuación de PlANCK (1) y sustituir en ella las expresiones encontradas para la longitud de onda (3) y la energía necesaria para desplazar un electrón (5), se encuentra una expresión (6) que depende solamente de los medibles del experimento y constantes universales que están establecidas (i.e. la carga de un electrón $e$ y la velocidad de la luz en el vacío $c$ ). Con esta última expresión se facilitan las mediciones debido a las variables independientes y se mantiene la consistencia de que todos las variables manejadas son medibles factibles cuya incertidumbre es controlable.

$$
e \lambda V_{T}=h c
$$

\section{El haz de luz}

Se ha analizado la forma en que el LED irradia luz con el propósito de lograr el mejor resultado. En previas investigaciones se han utilizado LED pero no se presentan pruebas de consideraciones respecto a la radiación del LED (e.g. intensidad y distribución). En este estudio se consideró una distancia efectiva entre el LED y la rejilla. 


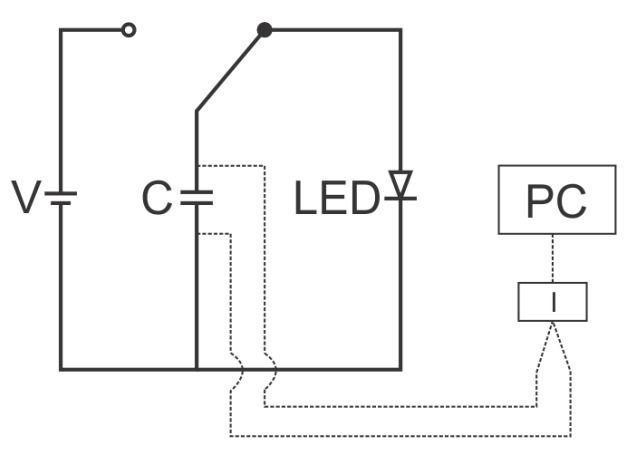

Figura 2: Diagrama del circuito utilizado y sus conexiones para medir el voltaje mínimo requerido por el LED. $(V)$ voltaje de la fuente, $(C)$ capacitancia, (I) interface junto con el sensor de voltaje.

Esto se realizó con el fin de obtener una mejor distribución de la radiación que pasa a través de la rejilla de difracción. Sugiriendo un análisis del ángulo de visión del LED, el cual consiste en la posición en donde la luz emitida por el LED se comporta o distribuye como un haz.

Esta posición o distancia efectiva para efectos del estudio, se consideró como un valor que se determina gradualmente como la distancia entre el LED y la rejilla hasta alcanzar un punto en donde los nodos difractados posean una proyección o apariencia más definida (Fig. 3 y 4 .

\section{Montaje}

Al trabajar con un LED que dividirá su haz de luz disminuyendo así la intensidad, es importante que el experimento se trabaje bajo poca iluminación. Lo cual mejora la observación de los nodos de interferencia constructiva eliminando perturbaciones en la definición de los nodos debido a la presencia de iluminación local. Sin embargo, al trabajar con poca iluminación se encuentra un problema, que es la poca visibilidad y consecuentemente poca capacidad de observar bien al momento de realizar las mediciones. Para evadir este inconveniente se propuso un montaje que considera el uso de una pequeña linterna, la cual iluminaría lo suficiente para poder realizar las mediciones sin dañar la proyección de los nodos (Fig. 3). Este montaje considera la forma en que el LED distribuye su luz y cumple a la vez con las dimensiones calculadas para obtener una incertidumbre baja.

Otra modificación al montaje fue la inclusión de una cámara para poder realizar las mediciones y registrarlas (Fig. 3). La cámara fue agregada por dos razones: primera, se trabajó con una rejilla que posee un gran número de divisiones (i.e. 570 divisiones por milímetro), lo cual hace que los puntos se separen entre sí, (con la cámara es posible ver los puntos simultáneamente) y segunda, la adición de un LED que emite luz infrarroja la cual sólo

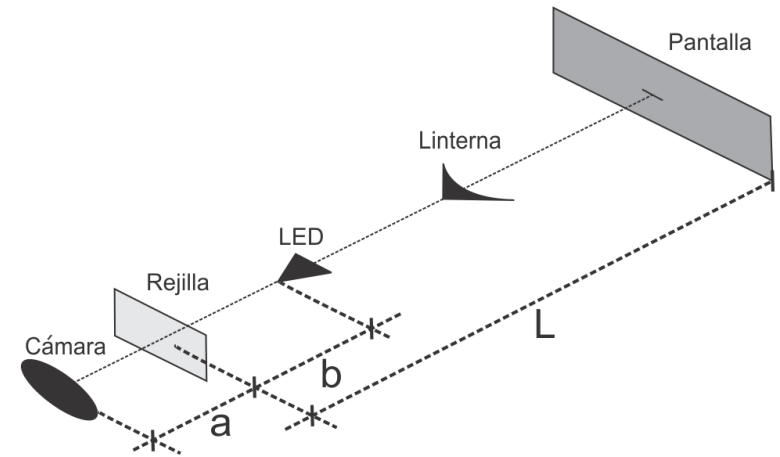

Figura 3: Diagrama del montaje necesario para la determinación de la longitud de onda.

es observable haciendo uso del sensor de la cámara [11].

Debido a la cantidad de componentes en el montaje fue necesario mantener el alineamiento entre cada uno de ellos ya que al no cumplir con este requerimiento se crearían alteraciones en el resultado. La influencia del alineamiento del montaje en el resultado del experimento es observable al momento de proyectar los nodos, ya que la falta de simetría entre ellos era el resultado de un mal alineamiento del montaje [11]. Un ejemplo de la proyección de los nodos se muestra en la figura 5 , en donde se observa la mejora en la medición basados en la claridad de los resultados. Esta metodología de medición había sido utilizada previamente en otros estudios [1] [4] obteniendo resultados satisfactorios.

Al momento de establecer el montaje se considera una distancia $b$ entre el LED y la rejilla. Esta es la distancia efectiva en donde el LED proyecta una mejor distribución de su iluminación, la cual se basa en el águlo de visión que éste posee (Fig. 44).

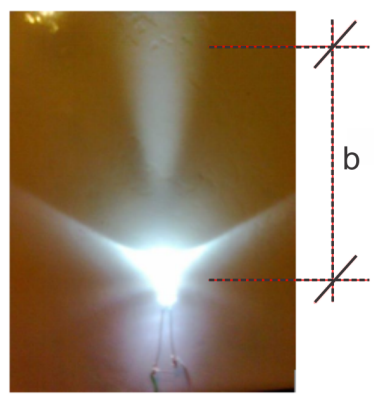

Figura 4: Distribución de la iluminación de un LED mostrando el áńgulo de visión.

\section{E. Resultados}

Como resultados de las mediciones se obtuvieron las dimensiones de las variables geométricas involucradas en el fenómeno (e.g. espaciamiento entre nodos $S$ y distancia 


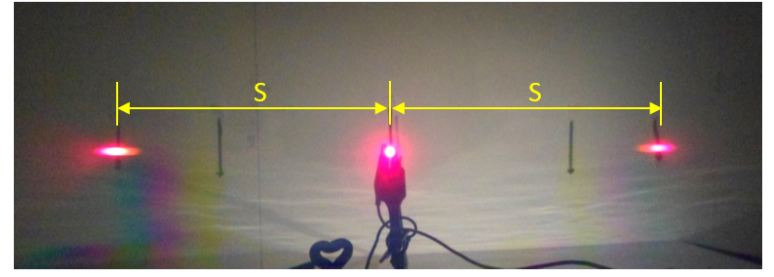

Figura 5: Captura de la cámara mostrando la metodología utilizada para medir la distancia de separación entre los nodos de interferencia constructiva necesarios para determinar la longitud de onda. El ejemplo muestra el uso de un LED Rojo.

entre rejilla y pantalla $L$ ). Estos resultados son presentados en la tabla 1, los cuales son necesarios para realizar los cálculos de las longitudes de onda de cada LED. Se consideró que la incertidumbre para la distancia $L$ sería de $5 \mathrm{~mm}$, estimando posibles errores en la localización exacta de la rejilla. Asimismo, se realizaron las mediciones de la descarga del capacitor para cada LED, haciendo uso de una interface. El resultado para cada descarga se ilustra en la figura 6, presentando el registro de datos hasta el momento en que el voltaje se comporta constante debido a que el LED deja de emitir luz.

Utilizando las ecuaciones 3 y 5 se determinó el valor del voltaje terminal y la longitud de onda para cada LED respectivamente (tabla2). Los valores para ambos resultados se encuentran dentro del intervalo de confianza de los valores esperados para cada LED [6]. Similitudes fueron encontradas entre LED que poseen el mismo color y provienen de diferentes fabricantes.

\begin{tabular}{lccc}
\hline LED & $L[\mathrm{~m}]$ & $S 1[\mathrm{~m}]$ & $S 2[\mathrm{~m}]$ \\
\hline Azul_01 & $1.500 \pm 0.005$ & $0.40 \pm 0.03$ & $0.40 \pm 0.03$ \\
Azul_02 & $1.500 \pm 0.005$ & $0.41 \pm 0.03$ & $0.41 \pm 0.03$ \\
Verde_01 & $1.500 \pm 0.005$ & $0.47 \pm 0.04$ & $0.47 \pm 0.04$ \\
Verde_02 & $1.500 \pm 0.005$ & $0.48 \pm 0.04$ & $0.48 \pm 0.04$ \\
Amarillo_01 & $1.500 \pm 0.005$ & $0.52 \pm 0.04$ & $0.52 \pm 0.04$ \\
Amarillo02 & $1.500 \pm 0.005$ & $0.53 \pm 0.04$ & $0.53 \pm 0.04$ \\
Rojo & $1.500 \pm 0.005$ & $0.58 \pm 0.03$ & $0.58 \pm 0.03$ \\
Infrarrojo_01 & $0.700 \pm 0.005$ & $0.48 \pm 0.05$ & $0.48 \pm 0.05$ \\
Infrarrojo_02 & $1.500 \pm 0.005$ & $0.87 \pm 0.05$ & $0.87 \pm 0.05$ \\
\hline
\end{tabular}

Tabla 1: Mediciones obtenidas para cada LED. (S1) representa el extremo izquierdo del nodo de primer orden y (S2) representa el extremo derecho del nodo de primer orden, $(L)$ es la distancia entre rejilla y pantalla.

Con los resultados anteriores se realizó un ajuste de la tendencia de los datos logrando así calcular el valor de la constante de PLANCK. La ecuación 6 al ser la expresión definida para el diseño manejado, fue utilizada para hacer un ajuste lineal en donde la pendiente del ajuste lineal 7 es la constante de PLANCK.

$$
Y=e V_{T} ; \quad m=h ; \quad X=c / \lambda
$$

El resultado para el ajuste de datos que se obtuvieron se presenta en la figura 7, en donde el valor obtenido para

\begin{tabular}{lcc}
\hline LED & $V_{T}[\mathrm{~V}]$ & $\lambda[\mathrm{nm}]$ \\
\hline Azul_01 & $2.654 \pm 0.001$ & $(45 \pm 3) \times 10^{1}$ \\
Azul_02 & $2.662 \pm 0.001$ & $(46 \pm 3) \times 10^{1}$ \\
Verde_01 & $1.810 \pm 0.001$ & $(52 \pm 4) \times 10^{1}$ \\
Verde_02 & $1.899 \pm 0.001$ & $(54 \pm 4) \times 10^{1}$ \\
Amarillo_01 & $1.865 \pm 0.001$ & $(57 \pm 4) \times 10^{1}$ \\
Amarillo_02 & $1.880 \pm 0.001$ & $(59 \pm 4) \times 10^{1}$ \\
Rojo & $1.724 \pm 0.001$ & $(63 \pm 3) \times 10^{1}$ \\
Infrarrojo_01 & $1.133 \pm 0.001$ & $(98 \pm 8) \times 10^{1}$ \\
Infrarrojo_02 & $1.138 \pm 0.001$ & $(88 \pm 5) \times 10^{1}$
\end{tabular}

Tabla 2: Resultados obtenidos del voltaje terminal o voltaje de emisión para cada LED y sus respectivas longitudes de onda.

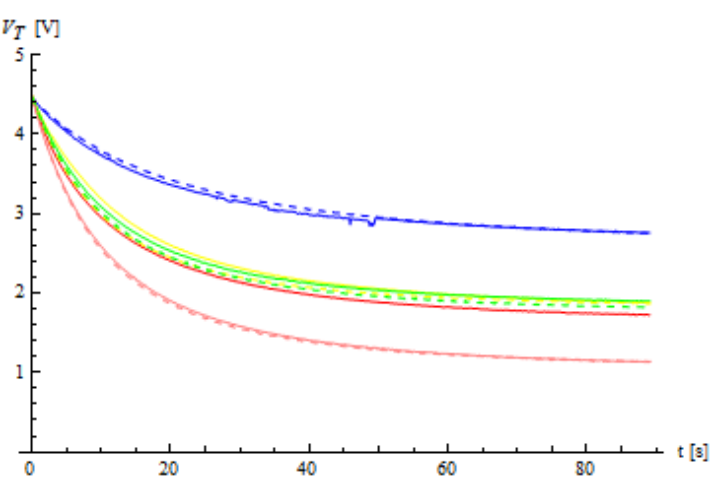

Figura 6: Serie de 800 datos registrados en 90 segundos para la descarga de los LED presentándolos con colores similares a los observados en las mediciones (infrarrojo es rosado). Las gráficas en línea discontinua muestra el decaimiento para los LED con código 01 y las líneas completas el decaimiento para los LED con código 02 tabla 1 .

la constante de PLANCK es de $(6.7 \pm 0.8) \times 10^{-34} \mathrm{~J} \cdot \mathrm{s}$ Este valor nos demuestra que se ha logrado alcanzar el orden de magnitud y la primera cifra significativa; la segunda cifra se considera dudosa. La incertidumbre de la medida resultó en un $12 \%$.

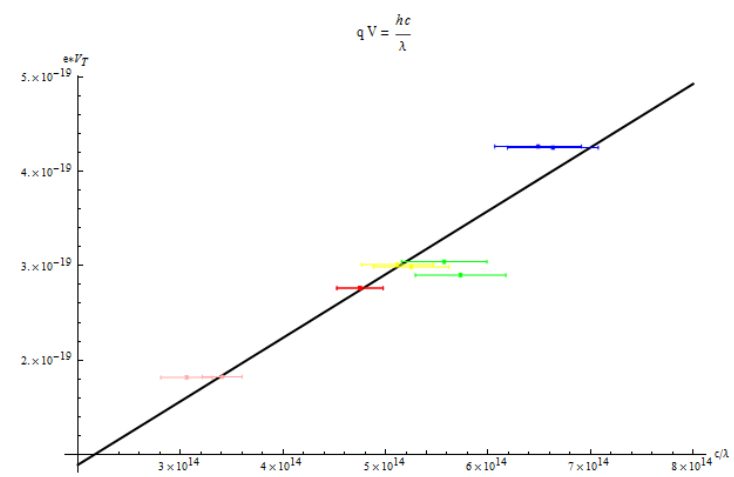

Figura 7: Ajuste lineal de los datos (la línea negra) de las mediciones de longitud de onda y voltaje terminal para cada LED con sus respectivas incertidumbres. Los LED se representan con los colores similares a los que muestran su emisión (El infrarrojo es rosado). 


\section{F. Discusión}

Basados en los resultados del experimento se determinó que el valor de la constante de PLANCK es $(6.7 \pm$ $0.8) \times 10^{-34} \mathrm{~J} \cdot \mathrm{s}$. Dicho valor, al ser comparado con el teórico $\left(h=6.626 \times 10^{-34} \mathrm{~J} \cdot \mathrm{s}\right)$, demuestra que el valor esperado esta contenido dentro de la incertidumbre del resultado. Por ende, se infiere que es un resultado exitoso por su exactitud al contener el valor esperado. De igual forma, posee una buena presición al tener un valor central aproximado al valor teórico. Sin embargo, se debe mencionar que la incertidumbre relativa de $12 \%$ hace de este resultado un valor poco preciso aunque exacto.

Una observación importante en el desarrollo del experimento es la descomposición de la luz emitida por los LED ya que al momento de realizar las mediciones se observó que los LED azul, amarillo y verde sufrían de descomposición espectral durante la difracción de luz. El LED azul se distinguí por presentar diversos tonos azules, desde celeste hasta morado (Fig. 8). Esto difiere de las especificaciones del fabricante quien ofrece el producto como LED de un color azul primario. Otros LED como el amarillo y el verde tenían un efecto diferente al descomponer su espectro durante la difracción, ellos variaban en una gamma de tres colores rojo, amarillo y verde, en donde cada uno de ellos se encontraba en magnitudes diferentes. Esto lleva a considerar que era posible que los datos hubieran sido incorrectos desde el momento de la medición, lo que puede interpretarse como una discrepancia en la tendencia obtenida (Fig. 7). La gama de colores era más notable al utilizar el LED verde, el cual mostraba los tres colores bien definidos (Fig. 8) a diferencia del LED amarillo el cual a pesar de sus especificaciones mostraba un color más rojizo o naranja. Estos nos sugiere que el material no necesariamente va actuar como se esperaría, lo cual conllevaría a errores en las mediciones, así como errores en procesos como el de la emisión de luz, en donde no necesariamente el voltaje encontrado es el voltaje esperado para el LED.

El hecho de tener diferentes gamas de colores para seis de los nueve LED utilizados se tomó en cuenta al momento de definir las incertidumbres de lasa separaciones $S$, en donde se utilizaron incertidumbres de $3-5 \mathrm{~cm}$. Estas incertidumbres sin duda alguna afectaron la incertidumbre del resultado final dada su dependencia. Sin embargo, ya que las incertidumbres no se involucran en el cálculo del valor central, se encuentra un valor preciso para el resultado de la constante de Planck. Por lo que se infiere que el alto valor de la incertidumbre relativa porcentual se debe a los altos valores asumidos par las incertidumbres de los medibles.

La precisión de este resultado se atribuye a las nuevas ideas aplicadas en las mediciones: La distancia efectiva entre el LED y la rejilla (ya que cada LED tiene su propio ángulo de visión) y el uso de la cámara para poder identificar con la sensibilidad de la misma la variación en la gama de los LED. Con esta comparación se encuentra que los LED de uso comercial no son fiables al momento de asumir sus propiedades físicas, lo que explicaría los errores en precisión encontrados en estudios anteriores.

Respecto al voltaje mínimo para que el LED irradie, se considera que se debe tomar no sólo cuando este encandece como un "punto" sino cuando este tiene un brillo "parejo" o en su forma técnica cuando este tiene la potencia necesaria con la cual se diseñó. Esta consideración se debe hacer ya que el voltaje decaía de forma indefinida muy por debajo del valor esperado. Se considera que para el modelo se debe utilizar la potencia con la cual el LED debe irradiar para ser observable al momento de hacer mediciones hasta alcanzar cierto voltaje estable.

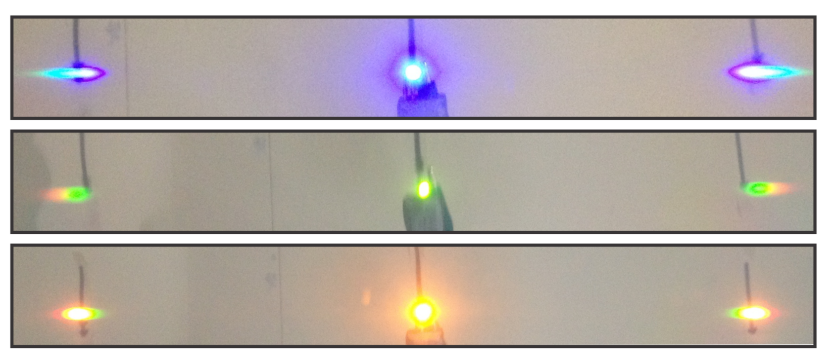

Figura 8: Gama de colores observados para los LED azul, verde y amarillo durante el fenómeno de difracción.

\section{G. Conclusiones}

En base a los resultados obtenidos, observaciones y comparaciones podemos concluir que el valor encontrado con este diseño experimental para la constante de PLANCK es $(6.7 \pm 0.8) \times 10^{-34} \mathrm{~J} \cdot \mathrm{s}$ el cual es aproximado al valor teórico de $6.626 \times 10^{-34} \mathrm{~J} \cdot \mathrm{s}$. El resultado posee una incertidumbre relativa del $12 \%$. Por lo que se concluye que el experimento logró un buen margen de exactitud demostrando ser eficaz aunque no muy preciso, lo cual se infiere como resultado de sobre estimar las incertidumbres.

Se encontró que el uso de la cámara para obtener una mejor observación del fenómeno mejora el resultado. En futuros estudios se sugiere el análisis de la radiación de un LED para determinar la localización y potencia necesaria, es decir la distancia efectiva entre el LED y la rejilla, y la intensidad con la que se maneja el LED, para tener un uso correcto y adecuado del mismo.

En general el uso de las nuevas metodologías para evitar la asunción de valores se considera como el camino adecuado a seguir; así como el buen uso de las dimensiones del montaje y el análisis de sus componentes tal y como se demostró en esta investigación. 


\section{REFERENCIAS}

[1] Etkina, E. y Planinsi, G. (2014). Light-emitting diodes: Exploration of underlying physics. The Physics Teachers, 52(212). Doi:10.1119/1.4868933.

[2] Foley, E.; Candela, D.; Martini, K. y Tuominen, M. (1999). An undergrauate laboratory experiment on quantized conductance in nanocontacts. Am. J. Phys., 1(67).

[3] Hecht, E. (1997). Óptica Capitulo 9 Interferencia. Tercera Edición (3th Ed.), Addison-Wesley.

[4] Kuhn, J. y Vogt, P. (2012). Diffraction experiments with infrared remote controls. iPhysicsLabs, The Physics Teachers.

[5] Nawrocki, W. (2008). Electrical and thermal conductance quantization in nanostructures. Journal of Physics: Conference Series, 129(1), 012,023. URL http://stacks . iop.org/1742-6596/129/i= $1 / \mathrm{a}=012023$

[6] Oksolar (2014), Technical led's led color chart. Recuperado de: http://http://www.oksolar.com/led/ led_color_chart.htm

[7] Serway, R. A. y Jewett, J. (2014). Physics for
Scientists and Engineers with Modern Physics. Brooks/Cole-Thomson Learning, Belmont.

[8] Soukiassian (2000). Measuring the conductance of gold atomic wires: quantized conductance of a break junction. Tesis, Purdue University, the Netherlands.

[9] Sunnu, J.; Satsanapitak, S.; Thamaphat, K.; Oopathump, C.; Bharmanee, P. y Limsuwan, P. (2012). Experimental set for measuring the planck's constant using led. The 15th International Conference of International Academy of Physical Science, 1(81), 33-36. Thailand.

[10] Tolley, R.; Silvidi, A.; Little, C. y Eid, K. (1993). Conductance quantization: A laboratory experiment in a senior level nanoscale science and technology course. Am. J. Phys., 1(81), 33-36.

[11] Vargas, L. (2014). Determinación de la longitud de onda de un led infrarrojo por medio de difracción. laboratorio avanzado ii. Universidad Nacional Autónoma de Honduras.

[12] Zhou, F. y Cloninger, T. (2008). Computer-based experiment for determining planck's constant using leds. The Physics Teachers, 46. 